\title{
Observation of end-vortex nucleation in individual ferromagnetic nanotubes
}

\author{
A. Mehlin, ${ }^{1}$ B. Gross, ${ }^{1}$ M. Wyss, ${ }^{1}$ T. Schefer,${ }^{1}$ G. Tütüncüoglu, ${ }^{2}$ F. Heimbach, ${ }^{3}$ A. Fontcuberta i Morral,${ }^{2}$ \\ D. Grundler, ${ }^{4}$ and M. Poggio ${ }^{1, *}$ \\ ${ }^{1}$ Department of Physics, University of Basel, 4056 Basel, Switzerland \\ ${ }^{2}$ Laboratory of Semiconductor Materials, Institute of Materials (IMX), School of Engineering, \\ École Polytechnique Fédérale de Lausanne (EPFL), 1015 Lausanne, Switzerland \\ ${ }^{3}$ Lehrstuhl für Physik funktionaler Schichtsysteme, Physik Department E10, Technische Universität München, 85747 Garching, Germany \\ ${ }^{4}$ Laboratory of Nanoscale Magnetic Materials and Magnonics, Institute of Materials (IMX) and Institute of Microengineering (IMT), \\ School of Engineering, École Polytechnique Fédérale de Lausanne (EPFL), 1015 Lausanne, Switzerland
}

(Received 8 November 2017; revised manuscript received 9 April 2018; published 23 April 2018)

\begin{abstract}
The reversal of uniform axial magnetization in a ferromagnetic nanotube (FNT) has been predicted to occur through the nucleation and propagation of vortex domains forming at the ends. We provide experimental evidence for this behavior through dynamic cantilever magnetometry measurements of individual FNTs. In particular, we identify the nucleation of the vortex end domains as a function of applied magnetic field and show that they mark the onset of magnetization reversal. We find that the nucleation field depends sensitively on the angle between the end surface of the FNT and the applied field. Micromagnetic simulations substantiate the experimental results and highlight the importance of the ends in determining the reversal process. The control over end-vortex nucleation enabled by our findings is promising for the production of FNTs with tailored reversal properties.
\end{abstract}

DOI: 10.1103/PhysRevB.97.134422

\section{INTRODUCTION}

The study of magnetization reversal in magnetic nanostructures is a topic of major fundamental and practical interest. In particular, a controllable, fast, and reproducible reversal is crucial for applications in high density magnetic storage. This process, however, is often conditioned by the presence of edge and surface domains. Near borders, magnetization tends to change direction in order to minimize stray field energy. As a result, the form of surfaces and edges-including any imperfections or roughness-determines the configuration of the magnetization in their vicinity. The resulting magnetization inhomogeneities tend to affect reversal by acting as nucleation sites for complex switching processes [1-5]. Furthermore, small differences in the initial configurations of edge and surface domains can lead to entirely different reversal modes, complicating the control and reproducibility of magnetic switching from nanomagnet to nanomagnet [6].

The high surface-to-volume ratio of magnetic nanostructures makes mitigating these effects essential in the design of high-density memory elements. Although the effect of roughness can never be completely eliminated, one way to reduce the influence of edges and surfaces on magnetic reversal is to use magnetic structures that support flux-closure magnetization configurations [7]. Since these configurations minimize stray field, edges and surfaces play a lesser role in determining both their equilibrium state and their dynamics than for nonflux-closure configurations. Ferromagnetic nanotubes (FNTs) are one type of nanostructure supporting such states, e.g., in the form of a global vortex state, in which all magnetization curls around the hollow core [8-12]. Reversal of uniform axial

*martino.poggio@unibas.ch configurations in FNTs has been predicted to occur through the nucleation and propagation of vortex configurations, which appear at the FNT ends [8,13-16]. Theory has so far only considered FNTs with perfect, flat ends, despite their importance as the nucleation sites of the vortex end domains. Measurements on ensembles of FNTs have revealed behavior consistent with vortex reversal modes, though results were complicated by inhomogeneities in the arrays and interactions between FNTs $[17,18]$. Recent experiments on individual FNTs have focused on the form of the stable magnetization configurations, rather than on the reversal mechanisms [9-12].

Here, we uncover the magnetization reversal behavior of individual FNTs. In particular, we show the experimental signatures of vortex end-domain nucleation in individual FNTs and reveal their dependence on the slant angle of the ends. Magnetization reversal in FNTs offers some potential advantages over the equivalent and well-understood process in ferromagnetic nanowires (NWs) [19-21]: In particular, the core-free geometry of FNTs has been predicted to favor uniform switching fields and high reproducibility [14,22,23]. Understanding and controlling the switching process in real FNTs is a crucial step toward enabling practical applications.

We study magnetization reversal in individual FNTs using dynamic cantilever magnetometry (DCM). This technique involves a measurement of the mechanical resonance frequency $f$ of a cantilever, to which the FNT of interest has been attached, as a function of a uniform externally applied magnetic field $\mathbf{H}$. The frequency shift $\Delta f=f-f_{0}$, where $f_{0}$ is the resonance frequency at $H=0$, reveals the curvature of the magnetic energy $E_{m}$ with respect to rotations about the cantilever oscillation axis [24,25]:

$$
\Delta f=\frac{f_{0}}{2 k_{0} l_{e}^{2}}\left(\left.\frac{\partial^{2} E_{m}}{\partial \theta_{c}^{2}}\right|_{\theta_{c}=0}\right),
$$


where $k_{0}$ is the cantilever's spring constant, $l_{e}$ its effective length, and $\theta_{c}$ its angle of oscillation. We simulate the DCM measurements by constructing a micromagnetic model of the experiment with the software package Mumax3 [26], which employs the Landau-Lifshitz-Gilbert micromagnetic formalism using finite-difference discretization. For each value of $H$, the simulations determine the equilibrium magnetization configuration and the second derivative of $E_{m}$ with respect to $\theta_{c}$, relating DCM signal to the magnetization configuration present in the FNT, as discussed in Appendix B. These insightscombined with the high torque sensitivity provided by ultrasoft Si cantilevers-provide a detailed picture of magnetization reversal in individual FNTs.

\section{SAMPLES}

FNT samples consist of a 30-nm-thick ferromagnetic shell of $\mathrm{CoFeB}$ surrounding a nonmagnetic GaAs core with hexagonal cross section. The amorphous and homogeneous $\mathrm{CoFeB}$ shell is magnetron sputtered onto template GaAs NWs [27], which are grown by molecular beam epitaxy on a $\mathrm{Si}$ (111) substrate using Ga droplets as catalysts [28]. During CoFeB deposition, a wafer of upright and well-separated GaAs NWs is mounted with $35^{\circ}$ angle between the long axis of the NWs and the deposition direction. The wafer is then continuously rotated in order to achieve a conformal coating. Scanning electron micrographs (SEMs) of the studied FNTs reveal continuous and defect-free surfaces, whose roughness is less than $2 \mathrm{~nm}$ [10]. The FNTs have a diameter, which we define as the diameter of the circle circumscribing their hexagonal cross section, between 270 and $300 \mathrm{~nm}$. Lengths from 0.6 to $2.9 \mu \mathrm{m}$ are obtained by cutting individual FNTs into segments using a focused ion beam (FIB) [9]. This procedure ensures FNTs with smooth and well-defined ends, which—in general-are

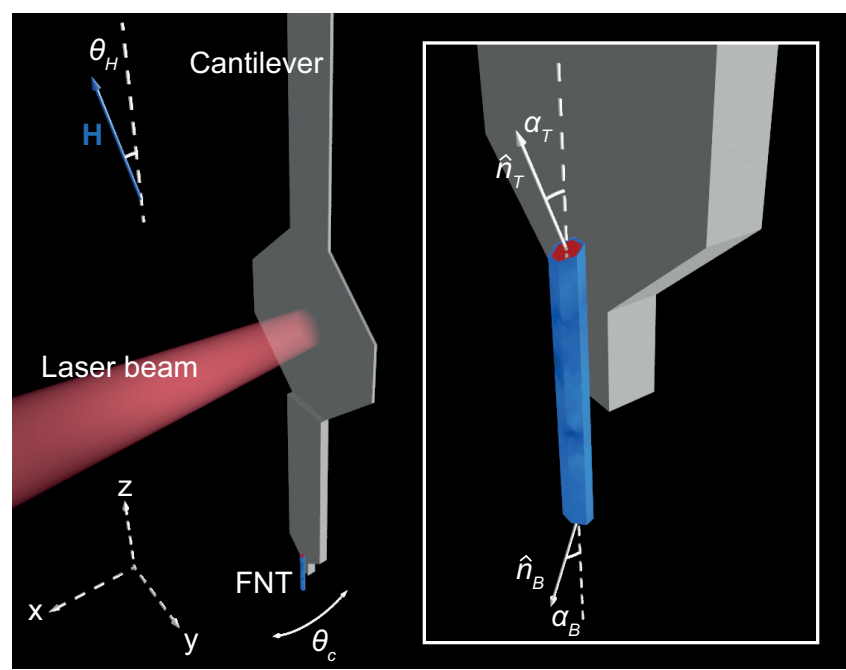

FIG. 1. Schematic diagram of the measurement: Si cantilever (gray) and CoFeB FNT (blue) with GaAs core (red). The cantilever oscillates about $\hat{y}$ and the FNT axis is parallel to $\hat{z}$. The applied magnetic field $\mathbf{H}$ can be rotated in the $x z$ plane by an angle $\theta_{H}$ with respect to $\hat{z}$. The unit vector $\hat{n}_{T}\left(\hat{n}_{B}\right)$ defines a perpendicular plane, in which the top (bottom) end of the FNT lies. The angle $\alpha_{T}\left(\alpha_{B}\right)$ refers to the angle of this vector with respect to the FNT axis $(\hat{z})$.

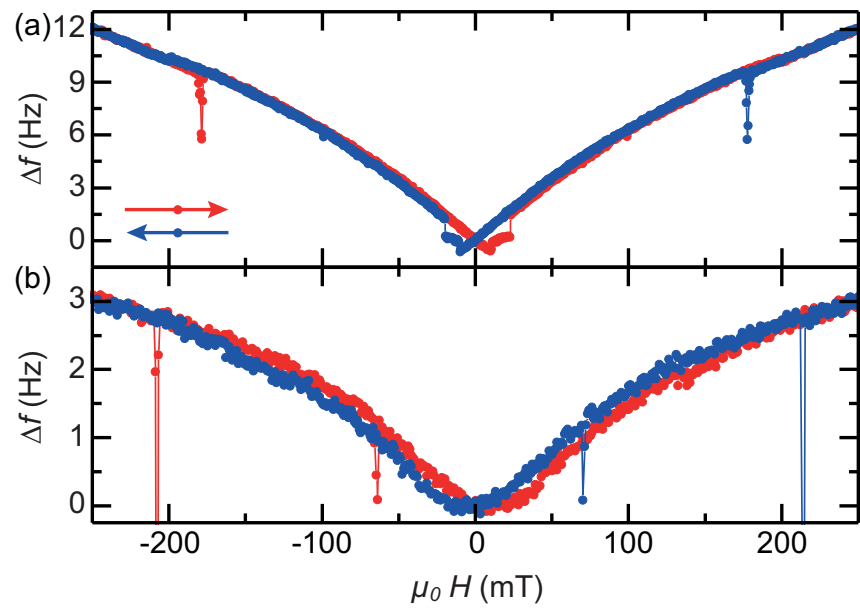

FIG. 2. Magnetic reversal of (a) a 2.2- $\mu \mathrm{m}$-long and (b) a 0.6- $\mu \mathrm{m}-$ long FNT measured by DCM at $280 \mathrm{~K}$. $\mathbf{H}$ is applied approximately along $\hat{z}$. As in all following figures, color-coded arrows denote the direction, in which the magnetic field is stepped.

tilted relative to the plane normal to the FNT axis. As shown in Fig. 1, we define the surfaces, in which these flat ends lie, using the normal unit vectors $\hat{n}_{T}$ and $\hat{n}_{B}$ for the top and bottom ends, respectively. We denote the angles of these ends with respect to the FNT long axis by $\alpha_{T}$ and $\alpha_{B}$. We use an optical microscope equipped with precision micromanipulators to pick up each FNT and affix it to the end of an ultrasoft Si cantilever, which is then mounted in the DCM measurement setup. Nonmagnetic epoxy (Gatan G1) is used as an adhesive.

\section{DCM MEASUREMENTS}

Figure 2 shows DCM measurements at $280 \mathrm{~K}$ of two FNTs of different lengths: (a) $2.2 \mu \mathrm{m}$ and (b) $0.6 \mu \mathrm{m}$. For each FNT, measured $\Delta f(H)$ is plotted for $\mathbf{H}$ applied approximately along its long axis $\hat{z}$ and stepped in the positive and negative direction. Since the cantilevers used here have similar mechanical properties, the magnitude of the frequency response is roughly proportional to the volume of magnetic material and therefore to the FNT length. Three major characteristics can be identified in the data sets. First, both show an overall V shape, consistent with magnetization reversal in a field $\mathbf{H}$, which is nearly coincident with the FNT easy axis [25]. Second, one or two spikes toward negative $\Delta f$ occur in forward applied field between \pm 220 and $\pm 60 \mathrm{mT}$ as well as weak echoes of these features in reverse magnetic field. Third, around zero field, where the slope of $\Delta f(H)$ inverts, a distinct difference between the two FNTs is evident. The shorter FNT shows a parabolic dependence, without ever becoming negative, while the longer one crosses to negative values of $\Delta f$ before exhibiting two discontinuous steps. The latter behavior is similar to that found for an even longer 2.9- $\mu \mathrm{m}$-long FNT. Measurements on FNTs of all three lengths were carried out at $4 \mathrm{~K}$ with similar results, shown and discussed in Appendix D.

\section{COMPARISON TO NUMERICAL SIMULATIONS}

The dimensions of a FNT are predicted to have a determining influence on its magnetic reversal. In particular, 


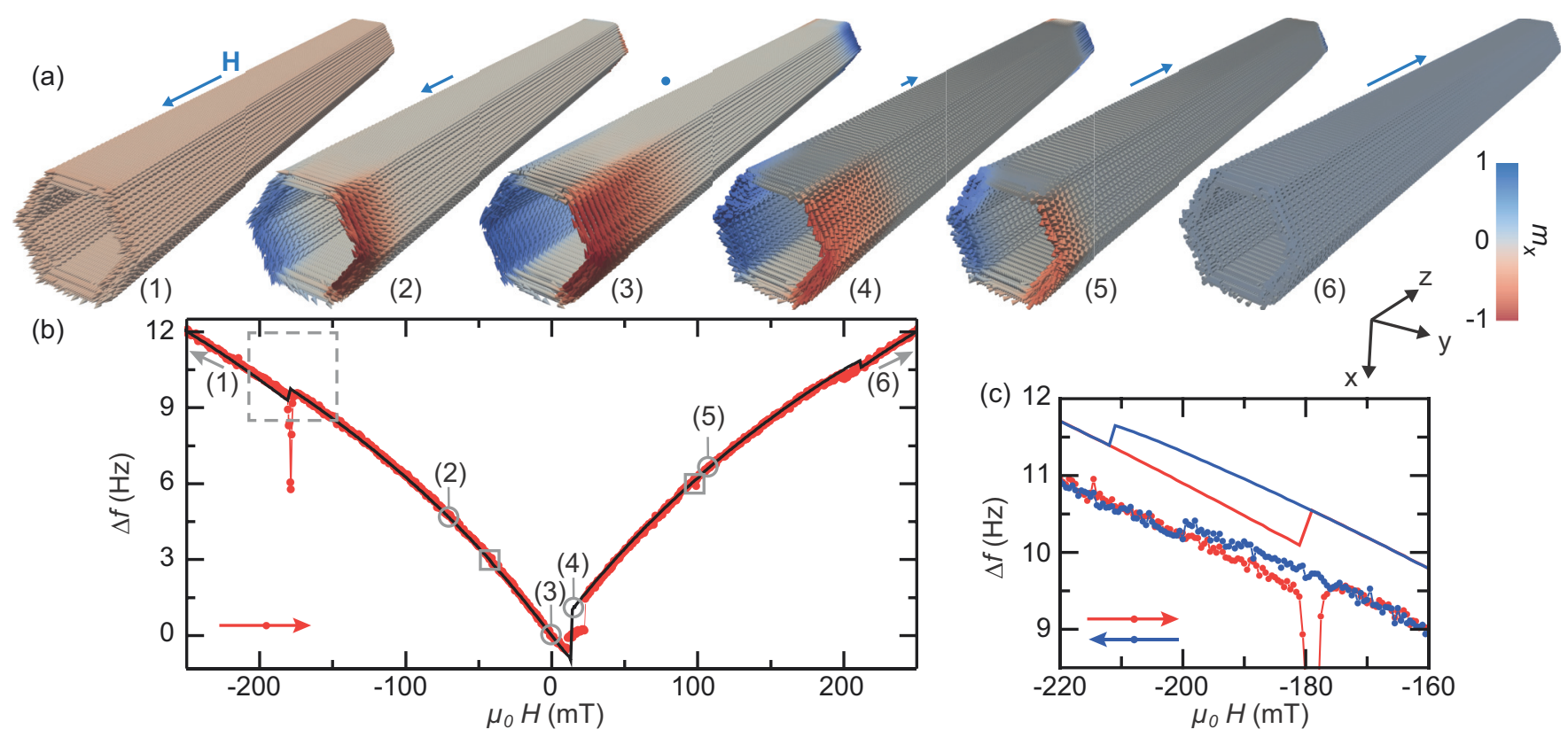

FIG. 3. Simulated and measured reversal of a 2.2- $\mu$ m-long FNT. (a) Simulated magnetization configurations for $\Delta f(H)$ corresponding to the labels. (b) Simulated (lines) and measured (points) DCM signal. Squares highlight those simulated vortex domain nucleation/annihilation features, which are difficult to see. (c) A detailed view of DCM signature corresponding to the nucleation (annihilation) of the first vortex domain [29]. The simulation, which is offset for clarity, uses $\alpha_{T}=6.5^{\circ}, \alpha_{B}=10.5^{\circ}$, and $\theta_{H}=11.0^{\circ}$.

FNTs with a larger than critical diameter reverse via nucleation of vortex rather than transverse domain walls $[8,16]$. Since this diameter ranges from a few nanometers to $20 \mathrm{~nm}$, all experimentally fabricated FNTs should reverse through vortex domains. For long FNTs, i.e., $2 \mu \mathrm{m}$ or longer for our cross-sectional geometry, the expected progression of the magnetization for $\mathbf{H}$ approximately along $\hat{z}$ can be summarized as shown in Fig. 3(a). This specific progression is the result of our simulations, but similar progressions were predicted by previous analytical and numerical models [8,25]. Starting from full saturation at negative $H$, vortex domains nucleate at the two tube ends, setting the nucleation field of the reversal. The nucleation field of each end vortex depends on the angle of the respective tube end with respect to $\mathbf{H}$, as discussed in detail in Sec. V. As $H$ approaches zero and becomes positive, the vortices grow along the tube axis toward the center. At a small positive reverse field, the magnetization in the central axial domain irreversibly inverts, while the vortex end domains persist. These vortex domains shrink in size with progressively larger positive $H$, until they are annihilated, marking the end of the reversal.

Figure 3(b) shows measured and simulated $\Delta f(H)$ for the 2.2- $\mu \mathrm{m}$-long FNT as $H$ is stepped in the positive direction. The simulated $\Delta f(H)$ is calculated using the measured properties of the cantilever and the geometrical and material parameters of the FNT (adjusted within their error). In Appendix C, we also consider the introduction of a magnetic dead layer near the FIB cuts, as reported previously [10,30,31]. Numerical labels indicate the magnetization configuration in Fig. 3(a) corresponding to a particular value of $H$ in Fig. 3(b). This correspondence allows us to attribute the discontinuous feature at $\mu_{0} H \approx-180 \mathrm{mT}$ in $\Delta f(H)$, between (1) and (2), to the nucleation of the first end vortex. The nucleation of the second end vortex, marked by a square between (2) and (3), though not visible in the measurement, produces a tiny step in the simulated response at $\mu_{0} H \approx-40 \mathrm{mT}$. Once at $H=0$, the FNT occupies configuration (3) with two vortex end domains and an axially aligned central domain. Between $\mu_{0} H \approx 10$ and $25 \mathrm{mT}$, an irreversible switching process causes the magnetization in the central domain to flip, forming configuration (4) and producing a change in the sign and slope of $\Delta f(H)$. The annihilation of the second end vortex between (4) and (5) can then be attributed to the discontinuity at $\mu_{0} H \approx 100 \mathrm{mT}$, while the first end vortex annihilates between (5) and (6) producing the feature at $\mu_{0} H \approx 200 \mathrm{mT}$.

In Fig. 3(c) we highlight the nucleation and annihilation of the first end vortex in both measured and simulated $\Delta f(H)$. The hysteresis marks the first and last irreversible processes of the magnetic reversal, indicating the nucleation and annihilation of the first end vortex, respectively. We find that both the magnitude in $\Delta f$ of the simulated vortex nucleation and annihilation features and the field, at which they occur, depend on the orientation of the FNT end surfaces (see $\hat{n}_{T}$ and $\hat{n}_{B}$ in inset to Fig. 1) with respect to $\mathbf{H}$. In the simulation, the angles of the ends with respect to the FNT long axis, $\alpha_{T}$ and $\alpha_{B}$, are carefully adjusted to match the measurements. For the 2.2- $\mu \mathrm{m}$-long FNT shown in Fig. $3 \alpha_{T} \neq-\alpha_{B}\left(\hat{n}_{T} \nVdash \hat{n}_{B}\right)$, resulting in two distinct pairs of nucleation and annihilation fields. One nucleation and annihilation pair is barely visible in both experiment and simulation due to that end's specific orientation with respect to $\mathbf{H}$, as discussed in Appendix F. These specific simulations do not reproduce the negative spike in the measurements at $\mu_{0} H \approx-180 \mathrm{mT}$, which may result from small geometric imperfections at the FNT ends. Further simulations, also discussed in Appendix F, show that while the nucleation and annihilation fields of the vortex end domains 




(1)

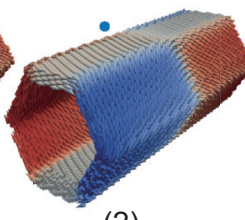

(2)

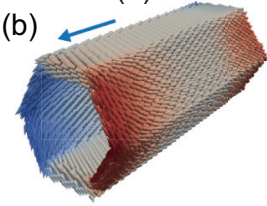

(c)

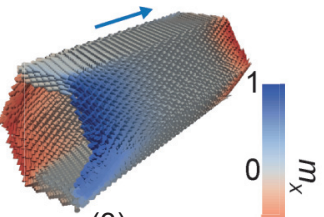

(3)
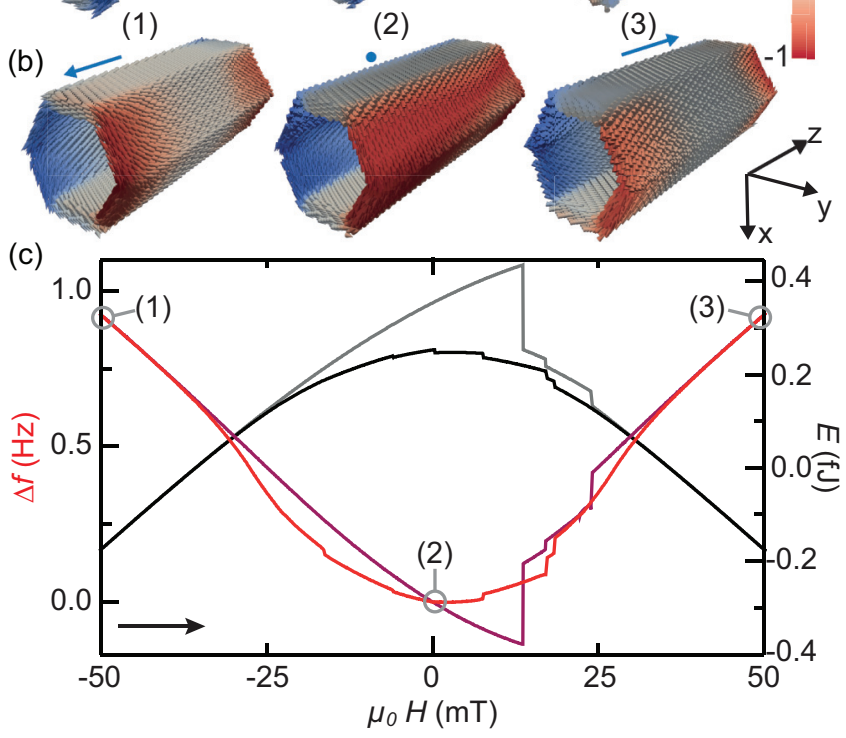

FIG. 4. Simulated reversal of a 0.6- $\mu \mathrm{m}$-long FNT. Equilibrium magnetization configurations corresponding to the labeled points in $\Delta f(H)$ for a FNT initialized with vortex end domains of (a) opposing and (b) matching circulation sense. (c) Plots of the simulated $\Delta f(H)$ in purple (red) and $E_{m}(H)$ in gray (black) for end domains of opposing (matching) circulation sense. For the simulation, $\alpha_{T}=6.0^{\circ}, \alpha_{B}=$ $10.0^{\circ}$, and $\theta_{H}=10.0^{\circ}$.

are robust to such defects, the form and magnitude of the corresponding DCM features can be strongly affected.

Although the overall features of the measured and simulated $\Delta f(H)$ for the 2.2- $\mu \mathrm{m}$-long FNT match, there is a difference in the irreversible switching of the central domain [around $\mu_{0} H \approx \pm 20 \mathrm{mT}$ in Fig. 3(b)]. The measured response shows two distinct steps interrupted by a plateaulike feature, rather than the single step predicted by the simulations. Measurements at slightly different $\theta_{H}$ and of the 2.9- $\mu$ m-long FNT result in one to three such plateaus in the switching region. These features indicate the presence of intermediate magnetization configurations near zero field. One such possible configuration is discussed in Appendix E.

For short FNTs-FNTs less than 2- $\mu \mathrm{m}$-long for our crosssectional dimensions-a different reversal process emerges. Since during reversal the two vortex end domains extend far enough to meet at the center of the FNT, the two relative circulation senses of the domains lead to two different progressions, shown in Figs. 4(a) and 4(b). For domains of opposing circulation sense, simulations show that after their nucleation, the central axial domain shrinks until only a domain wall remains to separate the two vortex domains $[32,33]$. As $H$ becomes increasingly positive, the axial wall reverses in a series of irreversible steps, which are associated with the replacement of the axial wall in two of the facets with a vortex and an antivortex wall. After full reversal of the axial wall, the vortex domains recede and annihilate.
For end domains of matching circulation sense, simulations show a progression, in which the two vortex domains merge at the center of the FNT without forming a domain wall. In reverse field, this global vortex configuration progressively rotates toward $\mathbf{H}$, until it splits and the resulting end vortices annihilate as the FNT saturates. Steps occurring during this rotation are associated with the switching of the magnetization in each hexagonal edge, where two facets meet.

In Fig. 4(c), we plot simulations of both $\Delta f(H)$ and the magnetic energy $E_{m}(H)$ associated with these two reversal progressions. Although vortex end domains with equal circulation represent the lower energy remanent configuration in short thin FNTs [9,34], both configurations have the same energy at high field within the accuracy of our simulation. Given the energy cost of switching between matching and opposing configurations, FNTs should—in principle—reverse via both reversal progressions. In fact, experiments on similar FNTs find both configurations in remanence after the application of an axial field [9]. Control over the relative circulation may be achieved by the introduction of structural asymmetries to the FNT ends [35,36]. Note that simulations plotted in Fig. 4(c) predict distinct $\Delta f(H)$ signatures for short FNTs with end domains of different relative circulation sense.

As shown in Fig. 5, the measured DCM response of the 0.6- $\mu \mathrm{m}$-long FNT matches the progression with vortices of matching circulation sense. $\Delta f(H)$ never drops below zero and is parabolic around zero field, indicating the presence of a remanent global vortex state. Features corresponding to the nucleation and annihilation of the vortex end domains appear at $\mu_{0} H \approx \pm 210 \mathrm{mT}$ and $\pm 70 \mathrm{mT}$ for the first and second vortices, respectively. These DCM signatures match the simulated response in field and to some extent
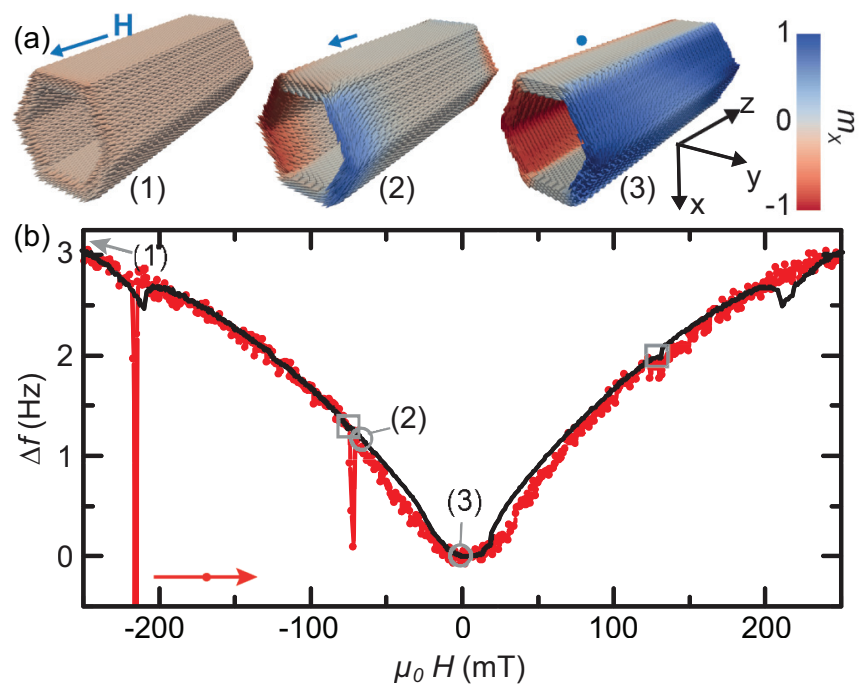

FIG. 5. Simulated and measured reversal of a $0.6-\mu \mathrm{m}-$ long FNT. (a) Calculated magnetization configurations for $\Delta f(H)$ corresponding to the labels. (b) Simulated (lines) and measured (points) DCM response. Squares highlight those simulated vortex nucleation/ annihilation features, which are difficult to see. For the simulation, $\alpha_{T}=4.0^{\circ}, \alpha_{B}=6.5^{\circ}$, and $\theta_{H}=10.0^{\circ}$. 


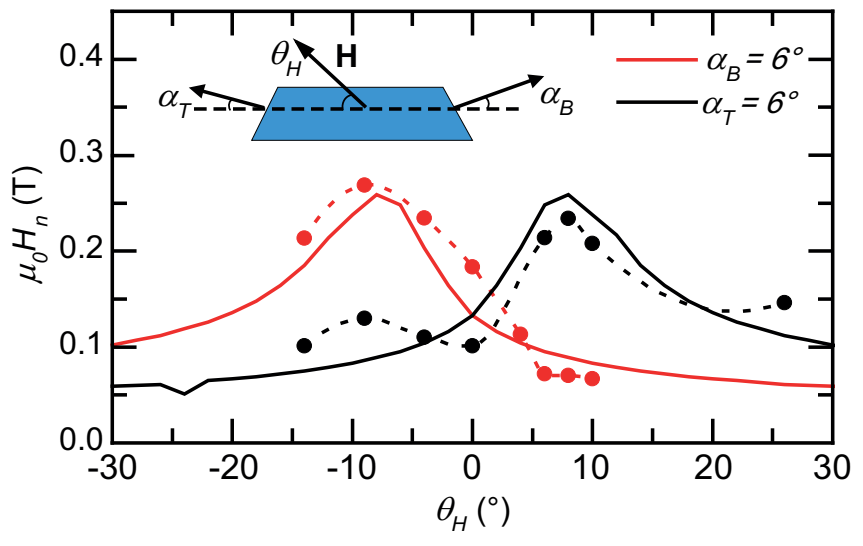

FIG. 6. Simulated and measured dependence of vortex nucleation field on field angle of a $0.6-\mu \mathrm{m}-$ long FNT. Black (red) points show the measured $H_{n}$ as a function of $\theta_{H}$ for the top (bottom) end vortex in a single FNT. The black (red) solid line shows the corresponding simulations for $\alpha_{T}=6.0^{\circ}\left(\alpha_{B}=6.0^{\circ}\right)$. The schematic diagram depicts the FNT, its angled ends, and $\mathbf{H}$.

also in $\Delta f$, with the exception of the spikes connected with the nucleation of the two vortices. This discrepancy is likely due to fine details of the end geometry not captured by our model.

\section{DEPENDENCE OF VORTEX NUCLEATION ON MAGNETIC FIELD ANGLE}

In all simulations, we tune the orientation of the plane in which the FNT ends lie $\left(\hat{n}_{T}\right.$ and $\left.\hat{n}_{B}\right)$ with respect to $\mathbf{H}$ in order to reproduce the measured features in $\Delta f(H)$ associated with vortex nucleation and annihilation. We study this dependence in more detail by measuring DCM in the $0.6-\mu \mathrm{m}$-long FNT as a function of $\theta_{H}$. Figure 6 shows the experimentally determined and simulated nucleation fields $H_{n}$ of the top (bottom) vortex domain in black (red) as a function of $\theta_{H}$. The corresponding annihilation fields, which are not shown, vary analogously. Measurements and simulations show that $H_{n}$ exhibits its absolute maximum just past $\theta_{H}= \pm \alpha_{T / B}$, i.e., $\mathbf{H} \| \hat{n}_{T / B}$. Upon a slight tilt of $\mathbf{H}$ away from this condition, $H_{n}$ is reduced. We attribute this behavior to the decrease in demagnetization field upon misalignment of $\mathbf{H}$. In a saturated FNT, the demagnetization field at the ends is maximized for $\mathbf{H} \| \hat{n}_{T / B}$. As a consequence, this alignment also maximizes the magnetic field $H_{n}$, at which the nucleation of a vortex domain is energetically favorable. As $\mathbf{H}$ and $\hat{n}_{T / B}$ are misaligned $\left(\theta_{H} \neq \pm \alpha_{T / B}\right)$, the demagnetization field at the ends decreases, resulting in smaller $H_{n}$. The DCM amplitude of the nucleation features also reduces sharply with this misaligment, as shown in Appendix F. This sensitivity to the end geometry likely explains why the strength of such features in DCM is often difficult to model and why vortex nucleation was not observed in previous experiments on FNTs, which had jagged or spherical ends [25].

The close agreement between experiment and simulation in Fig. 6 suggests that the simulated vortex nucleation process is an accurate description of what occurs in the measured samples. Further simulations, discussed in
Appendix $\mathrm{G}$, confirm that $H_{n}$-and therefore the reversal nucleation field - can be reduced from 250 to under $25 \mathrm{mT}$ by increasing the slant angle $\alpha_{T / B}$ from 0 to $30^{\circ}$ for a fixed orientation of $\mathbf{H}$.

\section{CONCLUSION}

We find that even slightly slanted ends considerably shift the nucleation field for axial magnetization reversal in FNTs. Still, the magnetization reversal process is observed to occur through vortex configurations, as originally predicted. A series of control simulations indicate that the reported behavior does not depend on the hexagonal cross section of the studied FNTs and should also hold for FNTs with cylindrical cross section. Our experimental confirmation of vortex-nucleated reversal and the demonstrated tunability of the vortex nucleation field set the stage for the realization of nearly ideal FNTs, which promise to have fast and highly reproducible switching behavior.

\section{ACKNOWLEDGMENTS}

We thank S. Martin and his team in the machine shop of the Physics Department at the University of Basel for help building the measurement system. We acknowledge the support of Kanton Aargau, the Swiss Nanoscience Institute, the SNSF under Grant No. 200020-159893 and via sinergia network Nanoskyrmionics (Grant No. CRSII5-171003), the NCCR Quantum Science and Technology (QSIT), and the DFG via project GR1640/5-2 in SPP 153.

A.M. and B.G. contributed equally to this paper.

\section{APPENDIX A: MEASUREMENT SETUP}

The dynamic cantilever magnetometry (DCM) measurement setup consists of a vibration-isolated vacuum chamber with a pressure below $10^{-4}$ mbar. A separate manually rotatable superconducting magnet allows the application of an external magnetic field $\mathbf{H}$ up to $4 \mathrm{~T}$ in any direction in the $x z$ plane. We use cantilevers made of undoped single-crystal $\mathrm{Si}$ with a width of $4 \mu \mathrm{m}$ and a thickness of $0.1 \mu \mathrm{m}$. The cantilever used in DCM measurements of the 2.2- $\mu \mathrm{m}$-long FNT, which is shown in Fig. 7, is 180- $\mu \mathrm{m}$-long with $k_{0}=30 \pm 3 \mu \mathrm{N} / \mathrm{m}$

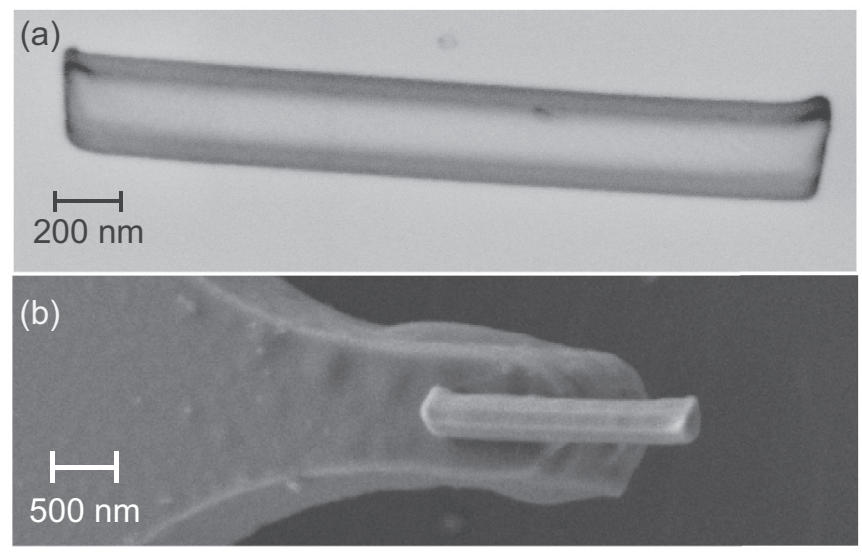

FIG. 7. Scanning electron micrographs (SEMs) of the FIB milled 2.2- $\mu$ m-long FNT (a) placed on a Si surface and (b) attached to the tip of an ultra-soft Si cantilever. 


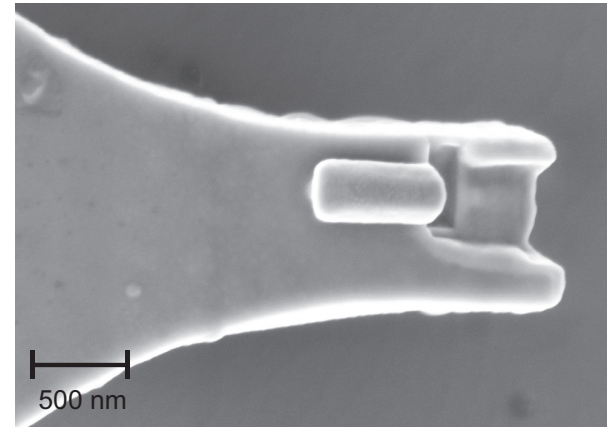

FIG. 8. SEMs of the 0.6- $\mu$ m-long FNT attached to the tip of a Si cantilever. The FNT was shortened in a second FIB step after the FNT was already attached to the cantilever.

and $l_{e}=130 \mu \mathrm{m}$. The cantilever used in measurements of the 0.6- $\mu \mathrm{m}$-long FNT, which is shown in Fig. 8, and of the 2.9$\mu \mathrm{m}$-long FNT is $150-\mu \mathrm{m}$-long with a spring constant of $k_{0}=$ $55 \pm 15 \mu \mathrm{N} / \mathrm{m}$ and an effective length of $l_{e}=105 \mu \mathrm{m}$. The large uncertainty in the determination of $k_{0}$-especially in the case of the $150-\mu \mathrm{m}$-long cantilever-is the result of spurious vibrational noise in our system that precludes a high fidelity measurement of the cantilever's thermal motion at different temperatures (the most reliable method for determining $k_{0}$ ). Deflection of the cantilever along $\hat{x}$ is measured using a fiber interferometer [37] with $100 \mathrm{nw}$ of $1550 \mathrm{~nm}$ laser light focused onto a $10-\mu \mathrm{m}$-wide paddle near the end of the cantilever. A piezoelectric actuator mechanically drives the cantilever at its resonance frequency with a constant oscillation amplitude of $40 \mathrm{~nm}$ using a feed-back loop implemented by a field-programmable gate array. Self-oscillation enables the fast and accurate extraction of the resonance frequency $f$ from the cantilever deflection signal. Before measurement, we stabilize the temperature and fully magnetize the sample at large H. DCM data is then collected as the field is stepped toward zero and into reverse field at a maximum rate of $8 \mathrm{mT} / \mathrm{s}$.

\section{APPENDIX B: NUMERICAL SIMULATIONS}

All micromagnetic simulations reported here are carried out with Mumax3. We set $\mu_{0} M_{S}$ to its measured value of

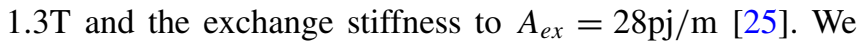
model the FNTs as perfectly hexagonal tubes with slanted ends. Discretization of space with cubic mesh elements leads to a staircase effect on all slanted surfaces, which could have an impact on the magnetic states that are calculated to be stable. In order to exclude spurious results due to such simulation artifacts, we perform additional reference simulations with the finite element package nmag [38], which avoids staircase effects by using irregular tetragonal meshes. In particular, nmag simulations reveal the same stable magnetization configurations, the same vortex nucleation mechanism, and the same values for the vortex nucleation (annihilation) field $H_{n}\left(H_{a}\right)$. As a result, we conclude that the staircase effect on the FNT ends does not have a significant effect on our simulation results.
TABLE I. Parameters used for the Mumax3 simulations shown in the main text. $d$ is the FNT diameter, $t$ its shell thickness, $l$ its length, $\alpha_{T}\left(\alpha_{B}\right)$ the slant angle of its top (bottom) end, $\theta_{H}\left(\phi_{H}\right)$ is the polar (azimuthal) angle of $\mathbf{H}, \delta \theta_{c}$ is the cantilever deviation angle used to calculate $\Delta f$, and $\epsilon_{m}$ is the mesh size.

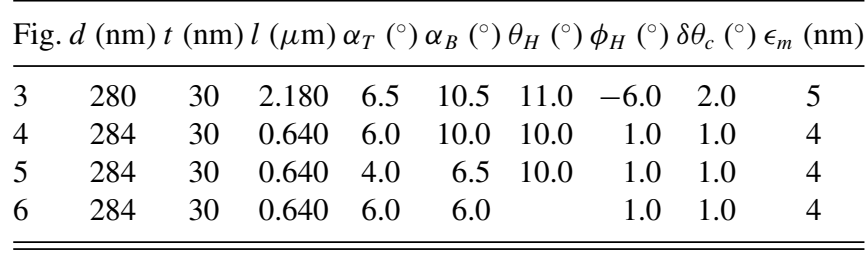

Both Mumax3 and nmag determine the equilibrium magnetization configuration for each external field value by numerically solving the Landau-Lifshitz-Gilbert equation. Since the microscopic processes in FNTs are expected to be much faster than the cantilever resonance frequency $[16,25,39,40]$, the magnetization of the nanotube is assumed to be in its equilibrium orientation. The calculation also yields the total magnetic energy $E_{m}$ corresponding to each configuration. In order to simulate $\Delta f$ measured in DCM, we numerically calculate the second derivative of $E_{m}$ with respect to $\theta_{c}$ found in (1). At each field, we calculate $E_{m}$ at the cantilever equilibrium angle $\theta_{c}=0$ and at small deviations from equilibrium $\theta_{c}=$ $\pm \delta \theta_{c}$. For small $\delta \theta_{c}$, the second derivative can be approximated by a finite difference: $\left.\frac{\partial^{2} E_{m}}{\partial \theta_{c}^{2}}\right|_{\theta_{c}=0} \approx \frac{E_{m}\left(\delta \theta_{c}\right)-2 E_{m}(0)+E_{m}\left(-\delta \theta_{c}\right)}{\left(\delta \theta_{c}\right)^{2}}$. By setting $f_{0}, k_{0}$, and $l_{e}$ to their measured values, we then arrive at the $\Delta f$ corresponding to each magnetization configuration in the numerically calculated field dependence. Table I shows the parameters used for simulations shown in each figure of the main text.

\section{APPENDIX C: DAMAGE INDUCED BY FIB CUTTING}

The cutting of the FNTs ends by FIB likely damages the $\mathrm{CoFeB}$ in the vicinity of the ends through the implantation of $\mathrm{Ga}$ ions. In particular, previous reports on the cutting of magnetic materials by FIB have found a magnetic 'dead layer' (a layer of material with strongly reduced or destroyed magnetism) near FIB cuts [30,31]. This dead layer has been found to be up to $200 \mathrm{~nm}$ in thickness depending on cutting parameters. In other work on similar FNT samples cut by the same process, we found that the dead layer extends around 100 $\mathrm{nm}$ from the ends [10].

In order to check the effects of such damage on the reversal process, we run a second set of simulations on all investigated FNTs using a reduced FNT length. For these simulations, we reduce the length near both cuts by $100 \mathrm{~nm}$. In all cases, the DCM signature and overall vortex nucleation behavior is unaffected by this length reduction, i.e., the shape of the DCM curve and the fields of the vortex nucleation and annihilation features do not change. However, the magnitude of the overall $\Delta f(H)$ signal is reduced due to the smaller FNT volume. Nevertheless, for both FNTs, quantitative agreement between the simulations with a reduced length and the measured signal is found by adjusting the spring constant $k_{0}$ of the cantilever within its uncertainty. Therefore, we conclude that the likely 


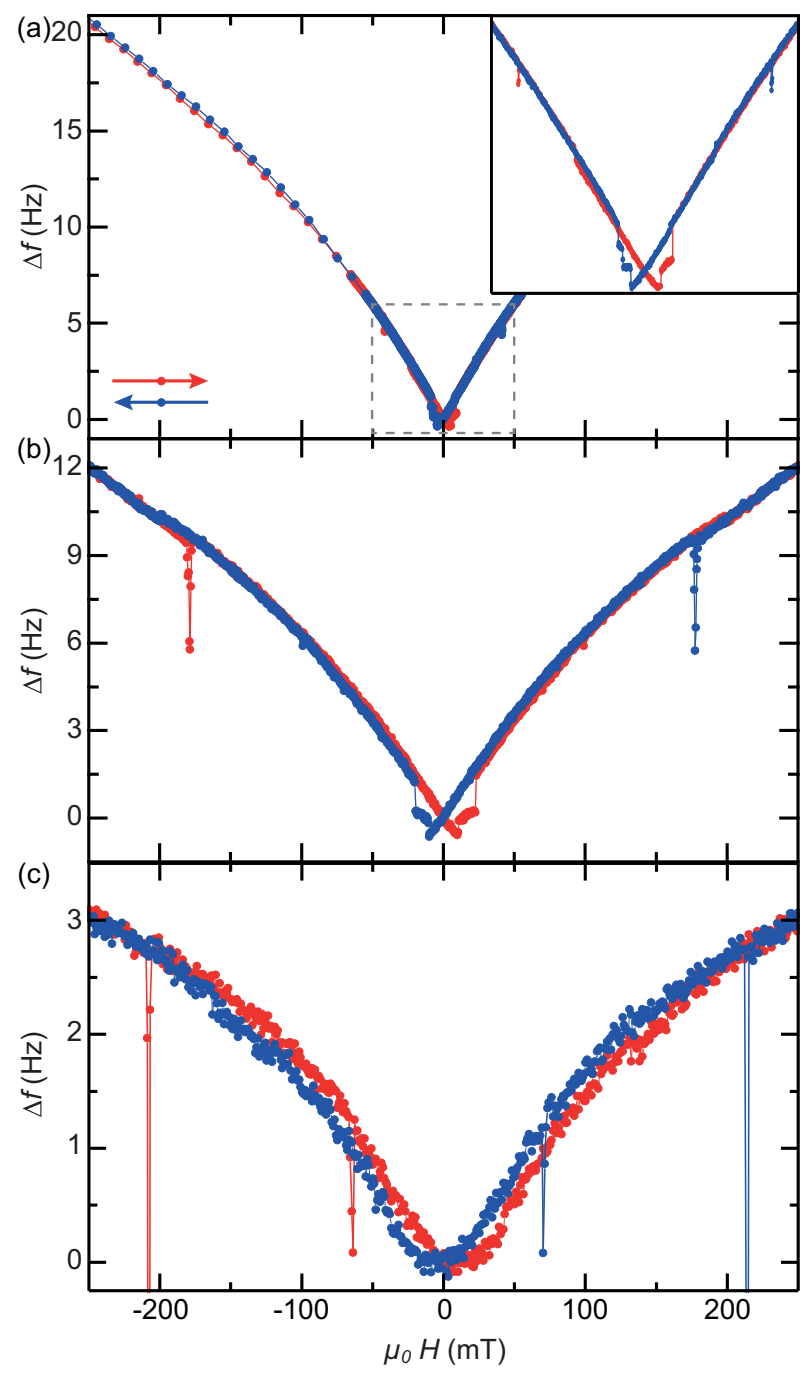

FIG. 9. Magnetic reversal of the three FNTs of different lengths measured by DCM at $280 \mathrm{~K}$ : (a) the $2.9-\mu \mathrm{m}$-long, including a zoom of the low field region; (b) the 2.2- $\mu \mathrm{m}$-long; and (c) the 0.6- $\mu \mathrm{m}$-long FNT.

damage induced by FIB cutting is not sufficient to change the vortex nucleation or reversal behavior in our FNTs.

\section{APPENDIX D: REVERSAL MEASURED AT HIGH AND LOW TEMPERATURE}

Figure 9 shows DCM measurements at $280 \mathrm{~K}$ of three FNTs of different lengths. Measurements of a 2.9- $\mu \mathrm{m}$-long FNT are shown in (a), along with measurements of the 2.2- $\mu \mathrm{m}$-long FNT and 0.6- $\mu \mathrm{m}$-long FNT, shown in (b) and (c), respectively. As expected from numerical simulations and theory, the FNTs longer than $2 \mu \mathrm{m}$ display a qualitatively similar behavior corresponding to a common magnetization reversal process.

Figure 10 shows a second set of DCM measurements of the same three FNTs carried out at $4 \mathrm{~K}$. Although the same qualitative features observed at $280 \mathrm{~K}$ can be recognized, various details of the reversal differ. First, the features in $\Delta f(H)$ indicating the nucleation or annihilation of a vortex

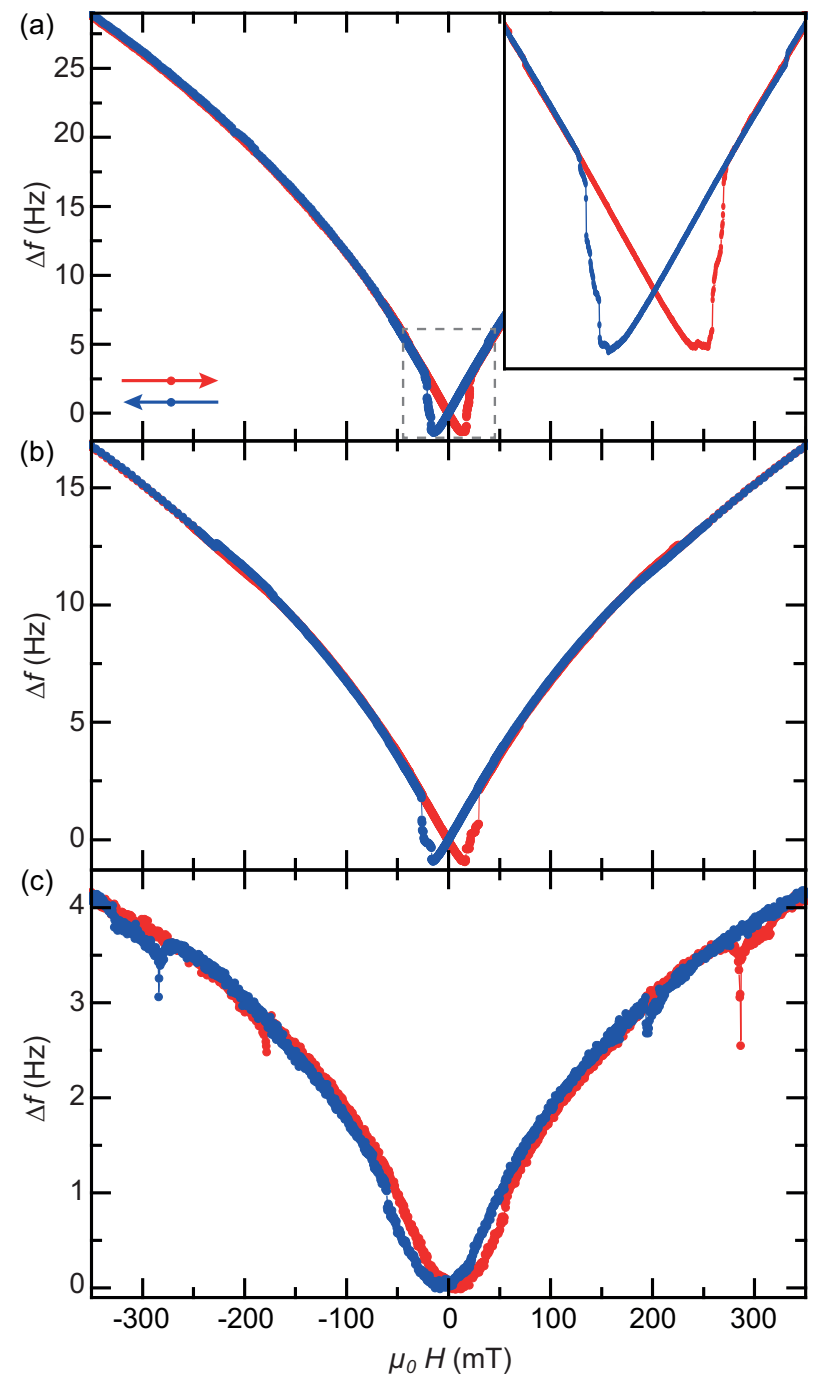

FIG. 10. Magnetic reversal of the three FNTs of different lengths measured by DCM at $4 \mathrm{~K}$ : (a) the 2.9- $\mu \mathrm{m}$-long, including a zoom of the low field region; (b) the 2.2- $\mu \mathrm{m}$-long; and (c) the $0.6-\mu \mathrm{m}$-long FNT.

are less pronounced at low temperature than at $280 \mathrm{~K}$. Second, for the two longer FNTs, the hysteric region marking an irreversible switching process spans a larger field range at low temperature than at high temperature. This behavior reflects the smaller amount of thermal energy available to the system at low temperature to overcome the energy barriers impeding magnetization reversal. Although the nucleation and annihilation of the two vortices appear at similar fields for all FNTs at both $4 \mathrm{~K}$ and $280 \mathrm{~K}$, differences in the angle of the magnetic field $\theta_{H}$ for each measurement preclude drawing conclusions about the dependence of nucleation/annihilation field on temperature.

\section{APPENDIX E: PLATEAU IN $\Delta f$ FOR SMALL APPLIED FIELDS}

DCM measurements shown in Figs. 2(a), 9(a), 9(b), 10(a), and 10(b) show plateaulike features in the irreversible switching region around zero field that are not predicted by the 

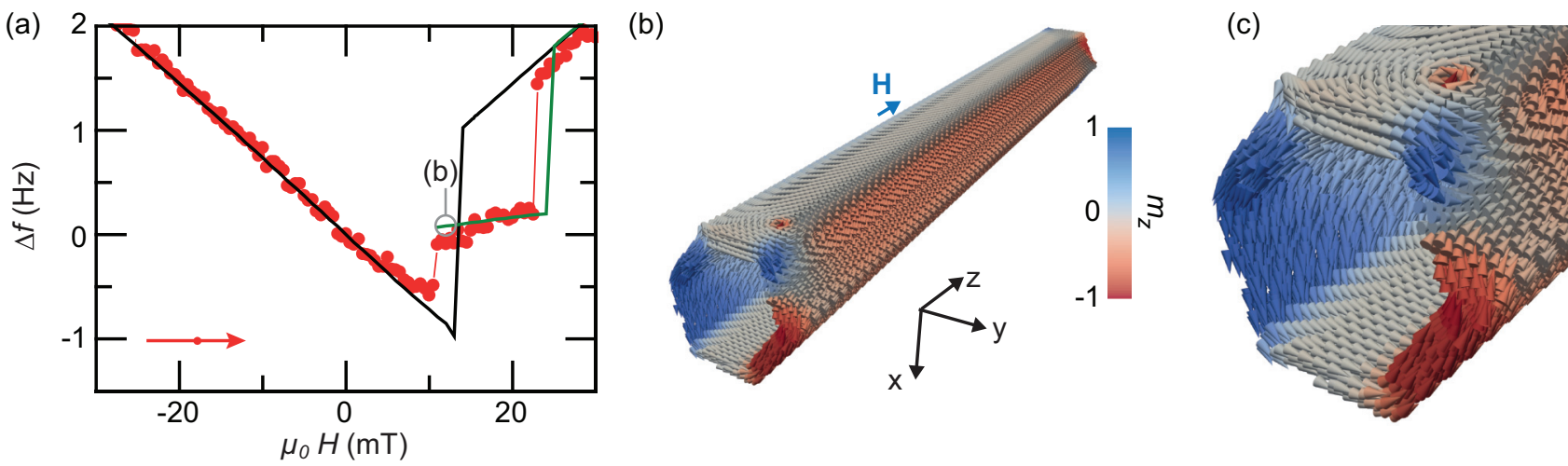

FIG. 11. (a) A detailed view of the simulated (line) and measured (points) DCM signal at low field, where the magnetization of the 2.2- $\mu \mathrm{m}$-long FNT irreversibly switches. The green line shows the simulated DCM response of a magnetization configuration, shown in (b) and (c), which is initialized and calculated to be stable at $H=0$. The configuration includes two vortices each residing in a hexagonal facet of the FNT and produces a $\Delta f(H)$, which matches the plateau appearing in the measured response.

simulations. This behavior in $\Delta f(H)$ can be reproduced, however, by initializing the FNT configuration at $H=0$. For example, by initializing the FNT with two vortices each residing in a hexagonal facet of the FNT and sweeping $H$ from zero to positive fields, a plateau in the simulated $\Delta f(H)$ emerges, as shown in Fig. 11(a). This feature corresponds to an intermediate configuration with two facet vortices, shown in Figs. 11(b) and 11(c). Following the irreversible switch around $25 \mathrm{mT}$, these facet vortices 'rotate' around $\hat{y}$ and take their place as the vortex end domains of an FNT in a mixed state configuration. In this picture, the irreversible switching of the FNTs central axial domain is characterized by the 'rotation' of end vortices into the facets-consuming the axial domain and resulting in the plateau in $\Delta f(H)$-followed by a second rotation of the vortices from the facets back to the ends. Similar states naturally occur in the simulations for short FNTs with opposing vortex circulation sense. Although the reversal mode described here matches the measured $\Delta f(H)$ near zero field, we cannot rule out the possibility of other intermediate configurations resulting in the same DCM response.

\section{APPENDIX F: SIMULATED AND MEASURED VORTEX NUCLEATION}

Figure 6 illustrates the dependence of the vortex nucleation on the magnetic field angle $\theta_{H}$. The plotted data are extracted from the DCM measurements shown in Fig. 12(b). Figure 12 shows a comparison between (a) simulated and (b) measured $\Delta f(H)$ in the region of one vortex nucleation for the $0.6-\mu \mathrm{m}-$ long FNT. Note the strong dependence of the magnitude in $\Delta f$ of the nucleation features on $\theta_{H}$. As with the value of $H_{n}$, this magnitude is maximized for $\mathbf{H} \| \hat{n}_{T / B}$. In other words, the vortex nucleation and annihilation features for an end whose normal is strongly misaligned with $\mathbf{H}$ are nearly invisible by DCM.

We carry out further simulations of vortex nucleation and annihilation in FNTs to test the effect of small geometric imperfections at their ends. Given the time required for each simulation and the large number of possible end imperfections, we simulate a few representative cases. In one case, we introduce a notch at one end of the FNT. The simulated notch cuts through the full thickness of the FNT, is 40-nm-wide along the
FNT circumference, and has length along the FNT axis ranging from 10 to $100 \mathrm{~nm}$. In Fig. 13, we show the simulated DCM feature related to vortex nucleation in that end with various

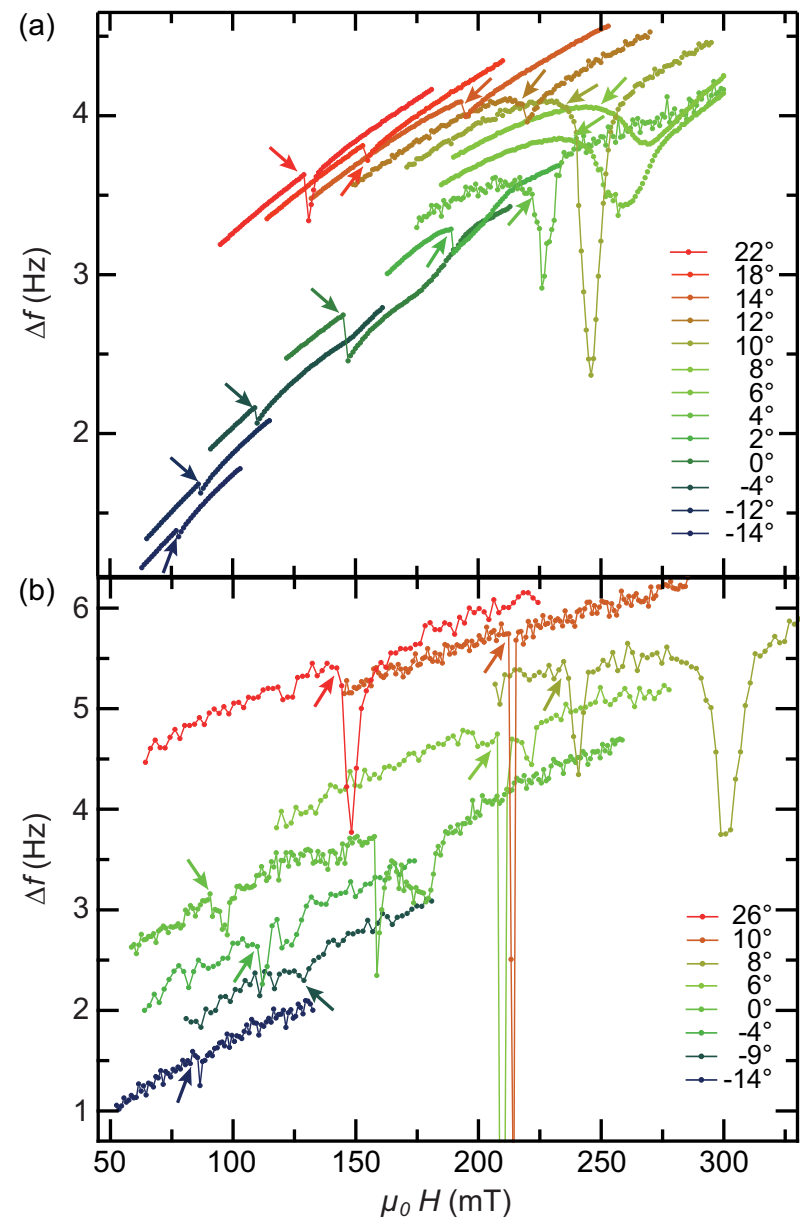

FIG. 12. (a) Simulated and (b) measured segments of $\Delta f(H)$ showing the vortex formation in a $0.6-\mu \mathrm{m}$-long NT for different values of $\theta_{H}$ as labeled in the plots. Arrows highlight the specific features corresponding to end-vortex nucleation. Here, $H$ is stepped from positive to negative values. 
(a)

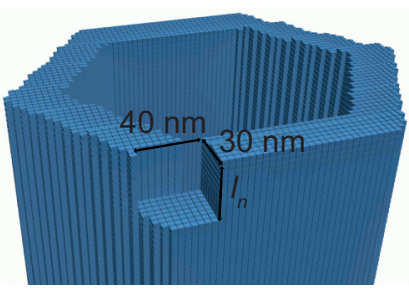

(b)

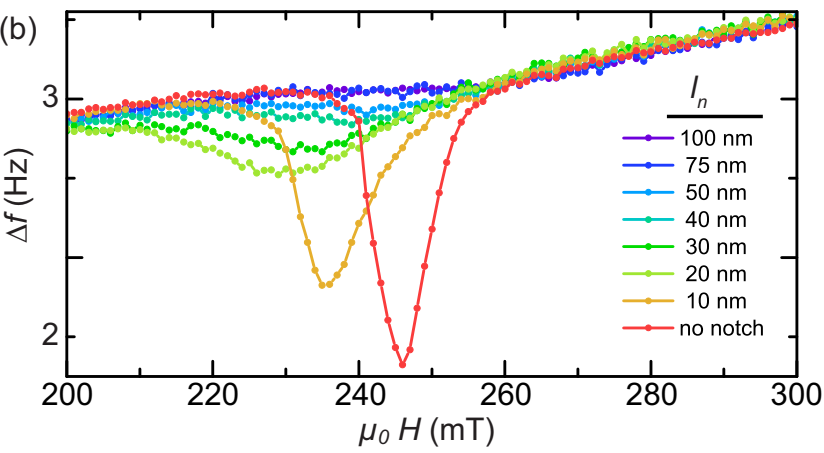

FIG. 13. (a) Schematic diagram of an FNT with a notch imperfection at its end. The notch cuts through the full 30-nm thickness of the FNT, is 40-nm-wide along the FNT circumference, and has length $l_{n}$ along the FNT axis. (b) Simulated segments of $\Delta f(H)$ showing vortex end-domain nucleation in a $0.6-\mu \mathrm{m}$-long NT. Simulations are shown for an FNT without a notch in its end and with a notch of various lengths $l_{n}$ as shown in the legend. Here, $H$ is stepped from positive to negative values.

notch lengths as well as without a notch. The simulations show that the dip in $\Delta f(H)$ related to the vortex nucleation is nearly eliminated by a notch longer than $30 \mathrm{~nm}$. Despite this strong reduction of the DCM feature, the simulations show that the vortex nucleation process and the magnetic field $H_{n}$, at which it occurs, remain nearly unchanged.

This simulation and others make clear that end imperfections of sizes which could be present in our samples (see Figs. 7 and 8), can strongly influence the form and magnitude of DCM

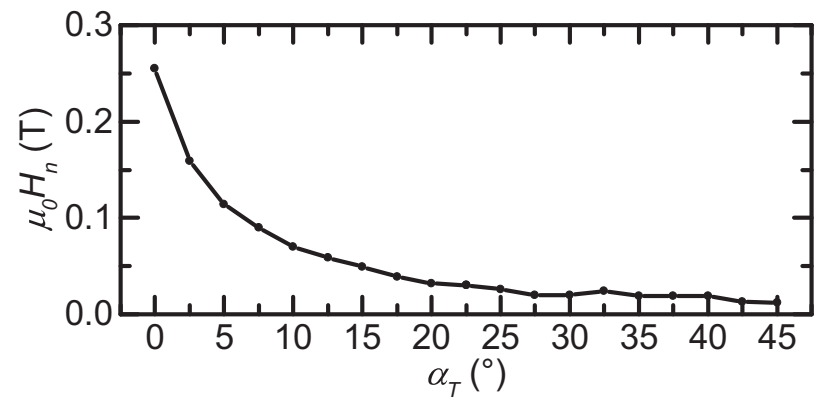

FIG. 14. Simulated dependence of the nucleation field $H_{n}$ of the top vortex on the slant angle of the top end $\alpha_{T}$ for the 0.6- $\mu \mathrm{m}$-long FNT. The magnetic field is applied parallel to $\hat{z}$, i.e., $\theta_{H}=0$.

features related to vortex nucleation and annihilation. In particular, such imperfections could explain why our simulations sometimes fail to reproduce strong spikes in measured $\Delta f(H)$ near the vortex nucleation or annihilation field. Despite this effect, geometric imperfections in this size range leave the nucleation and annihilation process itself nearly unaffected. These results provide a probable explanation for the mismatch in the exact form between the simulated and measured vortex nucleation features in $\Delta f(H)$ plotted in Figs. 3(c) and 5(b).

\section{APPENDIX G: CONTROL OF VORTEX NUCLEATION FIELD}

Figure 6 makes clear that the angle of the applied magnetic field $\theta_{H}$ affects the nucleation field of the vortex and thus the onset of magnetization reversal in the FNT. Figure 14 shows the simulated dependence of the nucleation field of the top vortex on the slant angle of the top FNT end $\alpha_{T}$ for a fixed $\theta_{H}$. The nucleation field can be tuned over 225 $\mathrm{mT}$ by changing the slant angle by $30^{\circ}$. These simulations, combined with the experimental evidence shown in the main text, show that reversal nucleation in FNTs can be finely and predictably controlled by tuning the geometry of their ends.
[1] J. M. D. Coey, Magnetism and Magnetic Materials (Cambridge University Press, Cambridge, 2010).

[2] T. R. Koehler and D. R. Fredkin, IEEE Trans. Magn. 27, 4763 (1991).

[3] T. Schrefl, J. Fidler, K. J. Kirk, and J. N. Chapman, J. Magn. Magn. Mater. 175, 193 (1997).

[4] J. Rothman, M. Kläui, L. Lopez-Diaz, C. A. F. Vaz, A. Bleloch, J. A. C. Bland, Z. Cui, and R. Speaks, Phys. Rev. Lett. 86, 1098 (2001).

[5] B. Hausmanns, T. P. Krome, G. Dumpich, E. F. Wassermann, D. Hinzke, U. Nowak, and K. D. Usadel, J. Magn. Magn. Mater. 240, 297 (2002)

[6] Y. Zheng and J.-G. Zhu, J. Appl. Phys. 81, 5471 (1997).

[7] A. D. Kent and D. L. Stein, IEEE Trans. Nanotechnol. 10, 129 (2011).

[8] P. Landeros, O. J. Suarez, A. Cuchillo, and P. Vargas, Phys. Rev. B 79, 024404 (2009).
[9] M. Wyss, A. Mehlin, B. Gross, A. Buchter, A. Farhan, M. Buzzi, A. Kleibert, G. Tütüncüoglu, F. Heimbach, A. Fontcuberta i Morral, D. Grundler, and M. Poggio, Phys. Rev. B 96, 024423 (2017).

[10] D. Vasyukov, L. Ceccarelli, M. Wyss, B. Gross, A. Schwarb, A. Mehlin, N. Rossi, G. Tütüncüoglu, F. Heimbach, R. R. Zamani, A. Kovács, A. Fontcuberta i Morral, D. Grundler, and M. Poggio, Nano Lett. 18, 964 (2018).

[11] M. Zimmermann, T. N. G. Meier, F. Dirnberger, A. Kákay, M. Decker, S. Wintz, S. Finizio, E. Josten, J. Raabe, M. Kronseder, D. Bougeard, J. Lindner, and C. H. Back, Nano Lett., doi: 10.1021/acs.nanolett.7b05222 (2018).

[12] Stano and F. Olivier, arXiv:1704.06614.

[13] N. A. Usov, A. Zhukov, and J. Gonzalez, J. Magn. Magn. Mater. 316, 255 (2007).

[14] P. Landeros, S. Allende, J. Escrig, E. Salcedo, D. Altbir, and E. E. Vogel, Appl. Phys. Lett. 90, 102501 (2007). 
[15] S. Allende, J. Escrig, D. Altbir, E. Salcedo, and M. Bahiana, Eur. Phys. J. B 66, 37 (2008).

[16] P. Landeros and A. S. Núñez, J. Appl. Phys. 108, 033917 (2010).

[17] J. Escrig, M. Daub, P. Landeros, K. Nielsch, and D. Altbir, Nanotechnology 18, 445706 (2007).

[18] J. Escrig, J. Bachmann, J. Jing, M. Daub, D. Altbir, and K. Nielsch, Phys. Rev. B 77, 214421 (2008).

[19] T. Wang, Y. Wang, Y. Fu, T. Hasegawa, H. Oshima, K. Itoh, K. Nishio, H. Masuda, F. S. Li, H. Saito, and S. Ishio, Nanotechnology 19, 455703 (2008).

[20] S. Vock, C. Hengst, M. Wolf, K. Tschulik, M. Uhlemann, Z. Sasvári, D. Makarov, O. G. Schmidt, L. Schultz, and V. Neu, Appl. Phys. Lett. 105, 172409 (2014).

[21] S. Jamet, S. Da Col, N. Rougemaille, A. Wartelle, A. Locatelli, T. O. Menteş, B. Santos Burgos, R. Afid, L. Cagnon, S. Bochmann, J. Bachmann, O. Fruchart, and J. C. Toussaint, Phys. Rev. B 92, 144428 (2015).

[22] Z. K. Wang, H. S. Lim, H. Y. Liu, S. C. Ng, M. H. Kuok, L. L. Tay, D. J. Lockwood, M. G. Cottam, K. L. Hobbs, P. R. Larson, J. C. Keay, G. D. Lian, and M. B. Johnson, Phys. Rev. Lett. 94, 137208 (2005).

[23] J. Escrig, P. Landeros, D. Altbir, E. E. Vogel, and P. Vargas, J. Magn. Magn. Mater. 308, 233 (2007).

[24] A. Mehlin, F. Xue, D. Liang, H. F. Du, M. J. Stolt, S. Jin, M. L. Tian, and M. Poggio, Nano Lett. 15, 4839 (2015).

[25] B. Gross, D. P. Weber, D. Rüffer, A. Buchter, F. Heimbach, A. Fontcuberta i Morral, D. Grundler, and M. Poggio, Phys. Rev. B 93, 064409 (2016).

[26] A. Vansteenkiste, J. Leliaert, M. Dvornik, M. Helsen, F. GarciaSanchez, and B. Van Waeyenberge, AIP Adv. 4, 107133 (2014).

[27] D. Rüffer, M. Slot, R. Huber, T. Schwarze, F. Heimbach, G. Tütüncüoglu, F. Matteini, E. Russo-Averchi, A. Kovács,
R. Dunin-Borkowski, R. R. Zamani, J. R. Morante, J. Arbiol, A. Fontcuberta i Morral, and D. Grundler, APL Mater. 2, 076112 (2014).

[28] F. Matteini, G. Tütüncüoglu, D. Mikulik, J. Vukajlovic-Plestina, H. Potts, J.-B. Leran, W. C. Carter, and A. Fontcuberta i Morral, Cryst. Growth Des. 16, 5781 (2016).

[29] See Supplemental Material at http://link.aps.org/supplemental/ 10.1103/PhysRevB.97.134422 for video VortexEntrance.avi showing the progression of simulated magnetization configurations as the applied magnetic field is swept through the nucleation field of a vortex end domain in the 0.6- $\mu \mathrm{m}$-long FNT.

[30] J. A. Katine, M. K. Ho, Y. S. Ju, and C. T. Rettner, Appl. Phys. Lett. 83, 401 (2003).

[31] C. O. Knutson, Magnetic domain wall dynamics in nanoscale thin film structures, Thesis, University of Texas (2008).

[32] A. P. Chen, N. A. Usov, J. M. Blanco, and J. Gonzalez, J. Magn. Magn. Mater. 316, e317 (2007).

[33] A.-P. Chen, J. M. Gonzalez, and K. Y. Guslienko, J. Appl. Phys. 109, 073923 (2011).

[34] A. P. Chen, K. Y. Guslienko, and J. Gonzalez, J. Appl. Phys. 108, 083920 (2010).

[35] M. Kläui, J. Rothman, L. Lopez-Diaz, C. A. F. Vaz, and J. A. C. Bland, Appl. Phys. Lett. 78, 3268 (2001).

[36] F. Giesen, J. Podbielski, B. Botters, and D. Grundler, Phys. Rev. B 75, 184428 (2007).

[37] D. Rugar, Appl. Phys. Lett. 55, 2588 (1989).

[38] T. Fischbacher, M. Franchin, G. Bordignon, and H. Fangohr, IEEE Trans. Magn. 43, 2896 (2007).

[39] T. Schwarze and D. Grundler, Appl. Phys. Lett. 102, 222412 (2013).

[40] M. Yan, C. Andreas, A. Kákay, F. García-Sánchez, and R. Hertel, Appl. Phys. Lett. 100, 252401 (2012). 